\title{
ANÁLISIS DEL LÉXICO DISPONIBLE DEL CENTRO DE INTERÉS DEL INSULTO EN ESTUDIANTES DE SECUNDARIA DE SAN LUIS POTOSÍ, MÉXICO
}

Marco Antonio Pérez Durán

\section{(9) $(\mathcal{P Q} \Theta \Theta$}

Doi: https://doi.org/10.15517/rfl.v46i1.41164

URL: https://revistas.ucr.ac.cr/index.php/filyling/index 



\title{
ANÁLISIS DEL LÉXICO DISPONIBLE DEL CENTRO DE INTERÉS DEL INSULTO EN ESTUDIANTES DE SECUNDARIA DE SAN LUIS POTOSÍ, MÉXICO
}

\author{
ANALYSIS OF THE AVAILABLE LEXICON IN A CENTER \\ OF INTEREST: INSULTS AMONG SECUNDARY SCHOOL \\ STUDENTS IN SAN LUIS POTOSI, MEXICO
}

\section{Marco Antonio Pérez, Durán}

\begin{abstract}
RESUMEN
El objetivo de este trabajo consiste en analizar las posiciones de los vocablos del centro de interés del insulto dentro de las conversaciones de estudiantes de secundaria de San Luis Potosí, México. Para tal fin, se analiza el índice de disponibilidad de los vocablos de 300 estudiantes por las variables sexo y centro educativo y se observa la posición que guarda el vocablo en la interacción. Los resultados indican que este centro acumula, entre las primeras respuestas, adjetivos, compuestos sintagmáticos y nominales.

Palabras clave: disponibilidad léxica; insulto; léxico disponible; socio-pragmática; vocablo.
\end{abstract}

\begin{abstract}
The aim of this article is to analyze the positions of the words of the center of interest of the insult within the conversations of high school students of San Luis Potosí, Mexico. For this purpose, the availability index of the words of 300 students is analyzed by the variables sex and educational center and the position that the word keeps in the interaction is observed. The results indicate that this center accumulates, between the first, adjective responses, syntagmatic and nominal compounds.

Keywords: lexical availability; insult; lexicon available; socio-pragmatic; word.
\end{abstract}

Dr. Marco Antonio Pérez Durán. Profesor investigador de tiempo completo de la Facultad de Ciencias Sociales y Humanidades. Universidad Autónoma de San Luis Potosí. San Luis Potosí, México.

Correo electrónico: marco.duran@uaslp.mx

Recepción: 22- 03- 19

Aceptación: 09- 07- 19 


\section{Introducción}

El objetivo de este trabajo consiste en analizar las posiciones de los vocablos de mayor índice de disponibilidad léxica (IDL desde este momento) del centro de interés del insulto dentro de las conversaciones de estudiantes de secundaria de San Luis Potosí capital ${ }^{1}$. Para tal fin, se analiza el IDL de los vocablos de la muestra, se presentan los resultados contrastivos de las variables sexo y centro educativo, y se observa la posición del vocablo en la interacción y su correlación con este índice.

El estudio de la disponibilidad léxica tiene su origen en los trabajos franceses de 1950. Su objetivo era claramente didáctico porque trataba de identificar el tipo de vocabulario que se utilizaba de manera más frecuente a partir de un tema. Después de 50 años, este método de trabajo sigue siendo primordial para el reconocimiento y obtención del léxico disponible de una comunidad cualquiera para contrastarlo y obtener una mejor descripción léxica. En lo que se refiere a la descripción del insulto, existe otro estudio "Temas de la disponibilidad léxica de la región NEA de argentina: mitos populares, la ciudad, el campo, insultos y diversiones nocturnas" del autor Wingeyer (2014), empero, su metodología es diferente al análisis que se hace, por lo que se espera robustecer la descripción hecha para este campo de trabajo tanto en el contexto internacional como nacional.

En la actualidad, la disponibilidad léxica presenta tres líneas de análisis claramente definidas, a saber: 1) como herramienta de otras disciplinas, 2) como herramienta para la planificación de primera y segunda lengua y 3) como método comparativo entre comunidades lingüísticas. Este trabajo se sitúa en el punto dos, para ello se utiliza el conocimiento acumulado de la socio-pragmática en la noción del léxico del insulto que está constituido por el vocabulario grosero y denigrativo. El primero hace referencia a la grosería como parte de la competencia léxica que puede o no usarse en una interacción, por ejemplo, 'puta', 'estúpido', 'idiota', etc. (este es el que se estudia en este trabajo); mientras que el segundo se refiere a cierto tipo de vocabulario que solo se identifica por el acto de habla. Este vocabulario es el más difícil de identificar porque no existe una expresión prototípica que demuestre denigración, puede ser cualquier palabra que presente una intencionalidad de agresión. Ambos tipos de vocabulario utilizan diferentes clases de expresiones que los informantes conocen y aplican para menospreciar, herir, sobajar o agredir en cualquier interacción comunicativa.

La disponibilidad léxica (DL desde este momento) tiene por objetivo recoger y analizar el léxico disponible de cualquier universo léxico. Cuando un hablante se encuentra inmerso en una situación comunicativa, este utiliza cierto tipo de vocabulario concreto que le ayudará a desenvolverse en ese tema (López Morales, 1995). Al léxico que se obtiene de ese tema, se le da el nombre de léxico disponible y se define como el conjunto de unidades léxicas que están a disposición del informante para un determinado momento comunicativo. Para obtener este léxico, se han propuesto 16 centros de interés acordes con la información léxica general de la comunidad, no obstante, desde hace tiempo se ha empezado a ampliar el número de centros para recolectar otro tipo de información que dé cuenta de la variedad de vocabulario de una lengua.

$1 \quad$ Este trabajo forma parte del proyecto de investigación Prácticas, discursos y reproducción sociocultural de la exclusión: discriminación y violencia escolar en secundarias rurales y urbanas de San Luis Potosí. Construcción de espacios escolares para mejorar la educación y las competencias, financiado por el CONACyT-189657, bajo la modalidad proyecto de investigación pedagógica. 
El insulto es prototípico de todas las culturas y de todos los contextos, porque en algún momento, sin importar edad, sexo o ideología, insultamos y cuando se hace, se esperan consecuencias devastadoras que afectan la individualidad y la colectividad (Ramírez, 1999). El insulto, para este trabajo, es una forma de defenderse y se define como el acto descortés que presenta el discurso en su uso, ya que utiliza un léxico claramente establecido y está relacionado al contexto de violencia. En México, por ejemplo, la violencia en el contexto educativo es un tema de actualidad por lo difícil que resulta hacer un estudio de este tipo que revele, de cierta forma, ¿cómo aparece?, ¿cómo se estructura?, ¿cómo afecta al sujeto amenazado?, ¿cómo se utiliza el vocabulario que sirve para ese fin? Lo único que se sabe es que UNICEF (2019) indicó que el $99 \%$ de los estudiantes ha sufrido o padecido algún tipo de violencia, pero no hay un dato revelador que hable sobre la violencia discursiva en las instituciones educativas del contexto nacional y, principalmente, local.

Solo se hace mención de los contextos sociales en el que la Secretaría de Educación Pública en 2007, por ejemplo, implementó el programa "Escuela segura", cuyo objetivo era convertir los centros escolares en espacios libres de violencia, delincuencia y adicciones. Sin embargo, ante la diversidad de tipos de violencia reconocidas (física, verbal, social, reproducida y de dispersión de rumores), la más difícil de analizar es la verbal, ya que se oculta entre la camaradería y la realidad agresiva de una comunidad (Ruiz y Muñoz, en prensa). La violencia discursiva consiste en utilizar el discurso como mecanismo de agresión, denigración y afectación emocional y su complejidad se basa en su espontaneidad y su dinamismo en el medio, se adapta y se modifica de acuerdo con las necesidades de los participantes (Rey y Ortega, 2008; Smith, 2004). Otro ejemplo de violencia es la que analizan Cerezo, Dolz, PonsSalvador y Cantero (1999) y Cava y Musitu (2002), quienes estudian la violencia reproducida que obedece a factores externos como la inseguridad en las calles, la venta de drogas, los asaltos, etc. y que se están reproduciendo en lo escolar. Esta se enfoca en la destrucción y el maltrato al recinto escolar como un mecanismo de desfogue emocional y contextual, en el que la sociedad impera sobre el comportamiento del sujeto.

Este trabajo está organizado en cinco apartados. En el primero, se lleva a cabo la descripción de la metodología desde la que se abordará este estudio. Posteriormente, se hace una breve exposición de la disponibilidad léxica y se describen, de forma escueta, algunos conceptos de la socio-pragmática para poder comprender el objetivo de este trabajo. Si bien es cierto que, en algunos momentos, la teoría analizada podría interpretarse erróneamente, se ha tratado de explicar lo más claro posible la información. En el tercer y cuarto apartado, se presenta el análisis de este centro en función de las variantes escuela y sexo, y se estudian los vocablos en las interacciones de estudiantes de secundaria. Al final del trabajo, se trata de dar respuesta al objetivo planteado en el apartado de conclusiones y discusiones.

\section{Metodología}

El corpus para el análisis corresponde a las respuestas de 300 estudiantes (150 hombres y 150 mujeres) de secundaria de centros educativos públicos y privados de la capital potosina y la muestra se obtuvo entre 2015 y 2016.

Como dato revelador, el Estado de San Luis Potosí ocupa el vigésimo segundo lugar como entidad federativa y el décimo quinto lugar en extensión dentro de la República Mexicana. Se encuentra en el centro-norte de México y cuenta con 58 municipios. De acuerdo con el análisis 
estadístico del Sistema Educativo Estatal (2016-2017) (SEP, 2017), el número total de centros educativos secundaria es de 1571 públicos y 124 privados. Por población, hay un total de 157 191 alumnos que cursan este nivel divididos en 78225 mujeres y 78966 hombres. Estos alumnos son atendidos por 9991 docentes. De acuerdo con la Encuesta Nacional de Victimización y Percepción sobre Seguridad Pública (INEGI, 2018), San Luis Potosí se encuentra entre las entidades federativas que incrementaron los índices de violencia en $63.2 \%$ entre 2013 y 2018 .

Las escuelas encuestadas pertenecen a centros educativos federales públicos y privados a los que asisten jóvenes entre los 11 y 16 años de edad de diferentes colonias de la capital potosina. Por ejemplo, una escuela secundaria se ubica en la colonia Nuevo Progreso, al sureste de la capital. Su índice de percepción de la violencia es alto y es una zona de migración hacia los Estados Unidos. La escuela tiene dos turnos de estudio - matutino y vespertino- y atiende en promedio a 800 estudiantes. En contraste, otra escuela de corte privado se ubica en el oriente de la capital, cuyo índice de percepción de la violencia es moderado. La diferencia es que en esta escuela solo hay un turno -el matutino- y el número de estudiantes es menor en comparación con la escuela pública.

En lo que se refiere al análisis de léxico disponible, se aplicaron al corpus las pruebas de disponibilidad léxica que consisten en recogida de datos, en el vaciado de estos y en su tratamiento computacional. Los estudios de esta área utilizan el cuestionario de disponibilidad léxica ${ }^{2}$, que es un detonante de la información y que está constituido por los centros de interés. Su finalidad es estimular el pensamiento del encuestado para obtener la información que se encuentra almacenada en la mente y traerla a la realidad lingüística en un tiempo determinado (dos o tres minutos). Las respuestas que se obtienen se les da el nombre de vocablo, el cual se opone al término palabra.

El vocablo es cada una de las unidades que componen el vocabulario. Es decir, los vocablos son las diferentes entradas léxicas que registra un diccionario de frecuencias, es más específico y restrictivo y es el resultado de la aplicación de criterios homogeneizadores o lematizadores (agrupación de todas las repeticiones de una palabra en una única entrada o vocablo) a las palabras o respuestas computables de los informantes en estudios de disponibilidad léxica; mientras que la palabra se refiere a todas y cada una de las unidades elementales, perfectamente distinguidas por la tipografía, que integran un corpus textual, escrito u oral (Cepeda et al., 2017 y López Rivero, 2008).

Una vez conseguidas las palabras, se hace la corrección tanto de contenido como de forma para, después, aplicar el tratamiento computacional en la que está integrada la fórmula matemática de López Chávez y Strassburger Frías (1991). De este procesamiento computacional, se obtienen el índice de disponibilidad léxica de los vocablos, la densidad léxica y el índice de cohesión analizados a partir de la posición en que aparecen las respuestas.

El índice de cohesión (IC desde este momento) se obtiene al dividir la media de palabras por informantes del centro por el número de vocablos que el centro recogió, la densidad léxica del centro (medida de repetición de palabra) se calcula dividiendo el número de sus palabras totales por el número de sus vocablos. Tanto la densidad como el índice de cohesión son factores que están influenciados por el número de informantes de la muestra, ya que el número de palabras diferentes aumenta conforme asciende el número de sujetos,

2 Cabe señalar que cada centro de interés se dispone tipográficamente en forma de columna, de modo que se observa el número de palabras por informante de una manera ágil y efectiva. 
es divisor en ambos cálculos (Hernández Muñoz, 2006, p. 311). Aunque Samper Hernández (2009, p. 131) rechaza categóricamente este dato en términos de promedio, porque no hay correspondencia de ninguna forma con la división exacta del número total entre los sujetos.

En la escala del reconocimiento, el ID comienza en el dato numérico 0. Este es el valor que establece que todas las palabras escritas por los encuestados son diferentes unas de otras durante el análisis de los listados y concluye con el valor numérico 1. Este valor establece que hay menos divergencia en las respuestas de los informantes por cada centro de interés. Todos estos datos estadísticos que se obtienen de la fórmula matemática de disponibilidad se aplican a sustantivos, verbos, adjetivos y compuestos sintagmáticos que, tradicionalmente, forman parte de las respuestas de esta área de estudio.

Uno de los problemas que se tuvieron que resolver fue el hecho de utilizar una metodología cuantitativa del tipo léxico-matemático a los estudios socio-pragmáticos sobre el insulto. Si bien es cierto que, tradicionalmente, se utilizan para los trabajos de este corte metodologías como: cuestionarios aplicados a la muestra, entrevistas, pruebas de percepción, entrevistas dirigidas o semi-dirigidas etc., también es cierto que, para este estudio, se utiliza la grabación anónima, solo como mecanismo de obtención de la información para visualizar la posición de los vocablos en la interacción, dejando a un lado su interpretación. Lo que se busca con este tipo de metodología es hacer la correlación entre los vocablos y su posición para determinar si el IDL es detonante de las posiciones en el texto.

La ventaja que presenta el uso de la grabación anónima es que genera una riqueza en cuestión de resultados, porque el sujeto, al no estar enterado de que está siendo grabado, restringe en menor medida su comportamiento y su discurso, resulta más abierto y espontáneo en el uso de construcciones de este tipo. Aunque, en la actualidad, este método se considera inapropiado, por eso se exige un permiso de consentimiento por parte de los informantes para que sean utilizados sus datos. En este trabajo, se cuenta con los permisos de aprobación a través del proyecto. Lo complicado para obtener el léxico que se analiza ha sido la forma como se recolectó la información. Al ser una grabación anónima, no siempre se obtenían interacciones completas. La solución fue que se grabaron periodos seguidos de 0 a 30 minutos y, después, se procedía a revisar las grabaciones hasta encontrar aquellas conversaciones completas, tomando en cuenta que se buscaba el vocabulario de insulto (la grosería) en las interacciones. El resultado de esta metodología fue la obtención de doce conversaciones espontáneas -de una duración mínima de diez minutos y una máxima de veinte- de estudiantes de secundaria de San Luis Potosí capital, tomando en cuenta que los descansos tienen una duración de 30 minutos. Al contar con las grabaciones, se procedió a la transcripción de cada una de ellas, lo que generó en promedio 100 horas de trabajo. La nomenclatura utilizada en la descripción de las interacciones se presenta así: $\mathrm{H}$ para referirse a hombre y $\mathrm{M}$ para mujer, y va acompañada de números arábigos secuenciales, por ejemplo, H1, H2, H3... que indican la posición y participación de los informantes en las interacciones.

Como parte del análisis de las correlaciones del léxico disponible en las interacciones, se propusieron tres tipos de vocablos: apertura, estabilidad y cierre. A los vocablos que activan de cierta forma otros vocablos se les dará el nombre de vocablos de apertura. Son vocablos que poseen los índices más bajos y, conforme transcurre la conversación, van cambiando de posición hasta llegar a ser vocablos estabilizadores. Su finalidad es mantener, como lo dice el término, la estabilidad durante el proceso de interacción. Se usan una y otra vez en repetidas ocasiones con el mismo significado y el mismo propósito. Su IDL se mantiene, pero la posición 
en la interacción cambia, lo que le permite al informante que los usa, aplicarlos correctamente. La mayoría de las conversaciones se llevó a cabo en la cafetería y en las canchas deportivas, lugares estratégicos para que los jóvenes se reúnan y puedan convivir abiertamente, sin la restricción de los profesores o prefectos. Los resultados de estas grabaciones se analizan en los siguientes apartados.

\section{Marco teórico}

Como es bien sabido, los léxicos disponibles son el reflejo del caudal léxico que los informantes usan para comunicarse en situaciones específicas y su aparición está determinada por la activación del tema (el insulto), y forma parte de las nuevas propuestas de ampliación y reconocimiento del léxico disponible ajenas al Proyecto Panhispánico en su estructura interna. Este centro se nutre más de adjetivos que de nominales y compuestos sintagmáticos, por lo que la diversidad de respuestas no está condicionada a una sola categoría gramatical.

La grosería se puede contabilizar y analizar en su posición, además, sus cambios se pueden observar desde un estudio longitudinal si así el estudio lo quisiera; no obstante, lo más destacable es que el vocabulario cambia y se actualiza, lo que puede ser hoy algo soez, mañana puede mostrar una carga de afectividad o de camaradería (Brenes Peña, 2007). En este contexto, la grosería desde el ámbito de la disponibilidad es 1) un vocablo al que se le puede contar y observar su posición dentro de la interacción; y 2) una estructura lingüística que puede estar conformada por una palabra: 'puto', ‘pendejo', 'maricón', etc., o varias palabras que construyen un compuesto sintagmático: 'chinga tu madre', 'vete al diablo', 'hijo de tu pinche madre', etc. Así pues, cuando se estudia y se analiza un vocabulario como este en toda comunidad de habla, en cualquier idioma, su existencia forma parte de la comprensión del medio y la forma de insultar no está condicionada a ningún factor cultural, ni social, ni educativo. La gente insulta porque se siente amenazada y la única forma, antes de la agresión física, es una agresión verbal (Cava y Musitu, 2002). Por ello, al agredir, se insulta tomando como base los aspectos sociales más importantes para el afectado: familia, parentesco, identidad sexual, religión, etc.

Desde la socio-pragmática, el insulto está vinculado a la noción de la descortesía verbal, en oposición a la cortesía y en relación con la violencia lingüística, que se aprecia en la interacción. Para este trabajo, la cortesía es un conjunto de reglas que deben cumplir los individuos de una determinada sociedad, varía de una comunidad a otra y de una cultura a otra; además, es una marca de educación porque privilegia el diálogo por encima de la agresión, en tanto que todo lo que no se ajuste a esta regla será considerado descortés, prohibido, tabú o maleducado (Martínez Lara, 2009, p. 16).

Brenes Peña (2007) afirma que el término descortesía verbal es un comportamiento que emula elementos lingüísticos intrínsecamente agresivos y descorteses que se emplean en conversaciones prototípicamente coloquiales entre diferentes grupos sociales. Son estructuras lingüísticas encontradas en cualquier comunidad linguiística y cambia el significado de la grosería cuando se utiliza como medio de identidad. En este contexto, el insulto es un acto de habla que posee una forma lingüística y contiene un valor socio-pragmático con el cual se intenta agredir, atacar y humillar a una persona en un contexto de interacción, y usa la grosería como vehículo para ese fin (Gómez Molina, 2000, p. 103). Se llama contexto de interacción a la aparición de un vocablo en su situación comunicativa específica y se organiza como un espacio que configura la posición del vocablo en tres: inicial o de apertura, desarrollo y de término o conclusión de la interacción. 
De igual forma, en estos contextos se puede apreciar la relación cortés-descortés que, a su vez, se asocia a la idea coste-beneficio en la conversación, en la que se expresan el uso y la defensa de los argumentos como una estrategia de guerra entre los interactuantes, ellos saben que agreden a través de ciertas expresiones lingüísticas. En este sentido, la conversación se vuelve conflictiva y los participantes poseen una imagen pública o privada que deben defender en el medio (Lakoff, 1973). La imagen negativa consiste en el deseo del hablante de no sufrir imposiciones y de que sus actuaciones no se vean entorpecidas o impedidas por parte de los demás; por el contrario, la imagen positiva es el deseo de aprobación, estima y aprecio que los otros hacen de él.

Como ejemplo de esto, en los contextos laborales, los varones tienden en mayoría de las ocasiones a estar inmersos en relaciones descorteses con otros de su mismo género, en tanto que las mujeres no, ya que desde pequeñas se les educa socialmente a mantener en su diálogo la cortesía y a respetar los espacios en donde se ejecute la interacción (Ortega Salamanca y Vargas Cortés, 2017, pp. 83-103). Otro ejemplo es el contexto escolar en el que la diversidad de manifestaciones interactivas pone en evidencia la convivencia comunicativa. La grosería es productiva y no necesariamente agresiva, muchos investigadores (Calsamiglia y Tusón, 2002; Ortega Salamanca y Vargas Cortés, 2017) han acertado que, en los contextos juveniles, la tendencia sea a la camaradería, aunque Zimmermann (1996, p. 477) mencione que no existe, todavía, un amplio estudio sobre el insulto en la educación.

Las características de la descortesía verbal pueden ser: 1) una estructura lingüística dinámica y productiva en la lengua; 2) una expresión que se caracteriza por su tosquedad; 3) una estructura que genera un alto impacto de descortesía; 4) una estructura que es muy productiva en cualquier ámbito social: político, religioso, académico y por cualquier individuo; 5) una estructura lingüística que, desde lo socio-pragmático, atenta contra la imagen de la otra persona; y 6) una estructura que es interpretada como grosería, teniendo en cuenta lo que quiera dar a conocer.

La interacción es considerada un tipo de acto de habla que se utiliza para distintos fines comunicativos. El interlocutor es el encargado de proporcionar un valor positivo o negativo de este acto; en el trabajo, el interlocutor busca la ofensa y la desvaloración para resaltar la imagen negativa en la interacción o la imagen gremial. La teoría de los actos de habla es una teoría pragmática que se originó con la hipótesis de que la unidad mínima de lenguaje no solo tiene como función ser un enunciado o una expresión, sino además realizar determinados actos o acciones, como enunciar, plantear preguntas, dar órdenes, describir, explicar, disculpar, agradecer y felicitar, entre otros (Searle, 1990).

En lo que se refiere a las conversaciones de jóvenes, los rasgos lingüísticos (por así decirlo) más relevantes en sus interacciones son las expresiones descorteses y denigrantes que dañan, grosso modo, la imagen pública o privada en el contexto de la imagen social. Son expresiones que constituyen parte de una dinámica conversacional compleja, en la que solamente ellos presentan pleno conocimiento de los referentes y son expresiones que constituyen actos de transgresión a las normas de convivencia social (Ortega Salamanca y Vargas Cortés, 2017, p. 86).

Cuando se trabaja con vocabulario de este tipo, se tiene en cuenta que, como otros lexicones, se actualiza y se puede conocer la identidad social, cultural y lingüística, no solo del individuo, sino de toda la comunidad a través de su uso. En lo que refiere a esto, el insulto es prototípico de cualquier comunidad y, del contexto del que se obtiene la información, 
presenta una diversidad de respuestas acordes con la realidad en la que vivimos y en la que no se encuentra ajena la educación.

\section{Análisis de disponibilidad léxica}

En la Tabla 1 se recogen los resultados relativos al número de palabras aportadas por los informantes del centro de interés del insulto.

Tabla 1. Presentación de los datos cuantitativos del centro de interés

\begin{tabular}{|c|c|c|c|}
\hline Centro de interés & Palabras & $\begin{array}{c}\text { Media de palabras por } \\
\text { informante }\end{array}$ & Vocablos \\
\hline El insulto & 6489 & 21.63 & 356 \\
\hline
\end{tabular}

Se puede observar que el número de vocablos siempre es menor al de las palabras y este centro de interés ha obtenido 356 vocablos que representan el $5.75 \%$ de 6489 palabras. Esta cantidad se traduce en una media de 21.63 respuestas por informante. Los resultados del análisis que se hace por densidad léxica e índice de cohesión aparecen en la Tabla 2.

Tabla 2. Análisis de la DL y del IC de este centro de interés

\begin{tabular}{|c|c|c|}
\hline Centro de interés & Densidad léxica & Índice de cohesión \\
\hline El insulto & 18.22 & 0.060 \\
\hline
\end{tabular}

Tal como se observa, por índice de cohesión, este centro presenta 0.060 y, por densidad léxica, acumuló 18.22. Este dato permite comprender que el centro de interés no acumula entre las primeras diez respuestas el $75 \%$ de la totalidad, sino que presenta variación en los vocablos. Ahora, del análisis del cuestionario, en la Tabla 3 se presentan los 15 vocablos disponibles generales que se obtuvieron del procesamiento matemático.

Tabla 3. Los 15 vocablos generales más disponibles

\begin{tabular}{|l|l|c|}
\hline \multicolumn{1}{|c|}{ Vocablo } & IDL \\
\hline 1 & Puta & 0.517724 \\
\hline 2 & Pendejo & 0.502287 \\
\hline 3 & Puto & 0.436380 \\
\hline 4 & Estúpido & 0.425004 \\
\hline 5 & Güey & 0.421805 \\
\hline 6 & Idiota & 0.416969 \\
\hline 7 & Chinga tu madre & 0.328884 \\
\hline 8 & Zorra & 0.292375 \\
\hline 9 & Cabrón & 0.291049 \\
\hline 10 & Hijo de tu pinche madre & 0.272845 \\
\hline 11 & Maricón & 0.260222 \\
\hline 12 & Hijo de puta & 0.254321 \\
\hline 13 & Vete a la verga & 0.243221 \\
\hline 14 & Joto & 0.234591 \\
\hline 15 & Pícatelo & 0.223399 \\
\hline
\end{tabular}


Según la Tabla 3, los adjetivos ocupan varios lugares en la lista de los vocablos más disponibles: 'puta', 'pendejo', 'puto', ‘joto', 'estúpido', 'idiota' y 'cabrón'. Estos representan en esta lista el $46 \%$ de los vocablos disponibles. Los tres primeros lugares pertenecen a esta categoría gramatical que los informantes utilizan para generar el insulto. Le siguen algunos nominales en esta lista como 'güey'3 y 'zorra' que aparecen en los lugares 5 y 8 respectivamente y, por último, aparecen cinco compuestos sintagmáticos: 'chinga tu madre', 'hijo de tu pinche madre', 'hijo de puta', 'vete a la verga' y 'pícatelo', que representan el $44 \%$ de los 15 vocablos más disponibles.

En este análisis, la categoría gramatical más numerosa es el adjetivo con 159 (44.6\%), le siguen los compuestos sintagmáticos con 129 construcciones $(36.2 \%)$ y, en último lugar, se encuentran los nominales con 68 apariciones. En algunos estudios sobre los centros de interés, la aparición de adjetivos obedece a la complejidad del funcionamiento de estas unidades y a la consolidación de los adjetivos en las listas de disponibilidad léxica. En el trabajo de López y Torner (1992, pp. 23-45), por ejemplo, la selección del adjetivo casi siempre proporciona un número reducido de piezas léxicas que, además, se caracterizan por ser muy pobres en cuanto a la semántica. Sin embargo, el número de adjetivos que aparecieron presenta, en la muestra, un número elevado, lo que conlleva a determinar que el adjetivo es importante para la formación del insulto. Su tipo es valorativo porque destacan la información del discurso que se encuentra en su construcción y en la interacción, además son adjetivos que van apareciendo para matizar, valorar o ponderar la información que los estudiantes van introduciendo al discurso descortés. En ese sentido, este centro ha de considerarse uno que acumula entre las primeras respuestas más adjetivos que otra categoría gramatical.

En el análisis por la variable sexo, la muestra se dividió en 150 hombres y 150 mujeres, de donde se obtuvo lo siguiente: los hombres generaron 3958 palabras de la muestra en tanto que las mujeres 2531 palabras. Cuando se describe un corpus con la misma cantidad de informantes en un estudio de disponibilidad léxica, el análisis contrastivo permitirá observar las variaciones sincrónicas de la lengua (Samper-Hernández, 2009). Para continuar con la descripción de los datos, ahora se presenta el análisis de media de palabra entre hombres y mujeres en la Tabla 4.

Tabla 4. Análisis de la media por la variable sexo

\begin{tabular}{|l|c|c|c|}
\hline & Palabras & $\begin{array}{c}\text { Media de palabras por } \\
\text { informante }\end{array}$ & Vocablos \\
\hline Hombre & 3958 & 20.83 & 176 \\
\hline Mujeres & 2531 & 16.87 & 180 \\
\hline Total & 6489 & 21.63 & 356 \\
\hline
\end{tabular}

Los resultados indican que los hombres presentaron 20.83 mientras que las mujeres 16.87, es decir, los hombres tienden a conocer más este vocabulario que las mujeres. Siguiendo con el análisis de los totales (356 vocablos), las mujeres presentan 180 vocablos disponibles

3 Si bien es cierto que el vocablo güey prototípicamente pueda desempeñar la misma función de tratamiento que boludo en Argentina o huevón en Chile y que no pueda ser considerado como insulto propiamente, en este trabajo se le considera como un vocablo que pertenece a este centro de interés y su uso está vinculado a la descortesía. 
que representan $50.5 \%$, mientras que los hombres alcanzaron 176 vocablos que corresponde a $49.5 \%$ de la totalidad de la muestra. A continuación, en la Tabla 5, se presentan los diez vocablos más disponibles:

Tabla 5. Los diez vocablos más disponibles por sexo

\begin{tabular}{|l|c|c|c|c|}
\hline & IDL & Hombres & IDL & Mujeres \\
\hline 1 & 0.4433 & Puta & 0.5256 & Pendejo \\
\hline 2 & 0.4242 & Pendejo & 0.3484 & Puto \\
\hline 3 & 0.3983 & Puto & 0.3403 & Puta \\
\hline 4 & 0.3924 & Estúpido & 0.3289 & Güey \\
\hline 5 & 0.3185 & Güey & 0.2902 & Estúpido \\
\hline 6 & 0.3101 & Idiota & 0.2855 & Chinga tu madre \\
\hline 7 & 0.2040 & Zorra & 0.2118 & Cabrón \\
\hline 8 & 0.1956 & Chinga tu madre & 0.2033 & Pinche \\
\hline 9 & 0.1785 & Hijo de tu pinche madre & 0.1887 & Zorra \\
\hline 10 & 0.1781 & Cabrón & 0.1821 & Idiota \\
\hline
\end{tabular}

De los datos obtenidos, las tres respuestas que aparecen en lo alto de la distribución del cuadro son iguales tanto para hombres como para las mujeres. Los vocablos 'puta', 'puto', 'pendejo', 'estúpido' y 'güey' son las respuestas más disponibles. El grado de coincidencia en las respuestas tanto para hombres como para mujeres es igual y, posiblemente, se debe a que estos vocablos se han generalizado en la comunidad linguiística. A partir de la posición número seis de la tabla comparativa surgen algunas diferencias significativas en cuanto al léxico disponible. Aparece un compuesto sintagmático en la posición ocho para hombres y seis para mujeres de las diez respuestas. Es extraño que aparezca un vocablo como 'zorra' en ambas listas, ya que, por lo general, es un vocablo exclusivo del insulto masculino al femenino.

En el análisis de los centros educativos públicos y privados, el número de palabras es de 2989 para centro educativo privado, que representa $46 \%$, y 3500 para el centro educativo público, que constituye $53.9 \%$ de 6489 palabras. Tal como se ha comentado, la muestra está dividida en 150 informantes por centro educativo público y privado que suman 300 . A continuación, se presenta la información en la Tabla 6.

Tabla 6. Análisis de la media por la variable centro educativo

\begin{tabular}{|l|c|c|c|}
\hline & Palabras & $\begin{array}{c}\text { Media de palabras por } \\
\text { informante }\end{array}$ & Vocablos \\
\hline Centro educativo privado & 2989 & 19.92 & 161 \\
\hline Centro educativo público & 3500 & 20.33 & 195 \\
\hline Total & 6489 & 21.63 & 356 \\
\hline
\end{tabular}

De lo anterior, el centro educativo público es muy productivo en la información del centro de interés del insulto. Esto se ve también reflejado en la media de palabras por informante que establece 20.33 por cada uno de ellos. Al depurar la muestra, se obtuvieron 195 vocablos producto de los 150 participantes del centro educativo público. Como se puede apreciar, la diferencia en vocablos entre un centro educativo y otro es de 34 vocablos y una 
diferencia de 511 palabras. Técnicamente, siempre se ha comentado que, en cuestión de agresión verbal, los estudiantes del centro educativo público son quienes más información poseen sobre el insulto, pero parece ser que la tendencia en el análisis es a la igualdad en todos los sentidos, la diferencia es mínima en los vocablos obtenidos en ambos centros educativos. En lo que a esto respecta, en la Tabla 7 se observan los diez vocablos más disponibles como en los criterios anteriores:

Tabla 7. Los diez vocablos más disponibles por centro educativo

\begin{tabular}{|l|c|c|c|c|}
\hline & IDL & Centro educativo privado & IDL & Centro educativo pública \\
\hline 1 & 0.7347 & Puto & 0.7336 & Chinga tu madre \\
\hline 2 & 0.5355 & Pendejo & 0.5456 & Pendejo \\
\hline 3 & 0.3849 & Chinga tu madre & 0.4835 & Puto \\
\hline 4 & 0.3078 & Güey & 0.3731 & Güey \\
\hline 5 & 0.2662 & Maricón & 0.3557 & Hijo de puta \\
\hline 6 & 0.2242 & Puta & 0.3463 & Vete a la verga \\
\hline 7 & 0.2187 & Cabrón & 0.3299 & Pinche \\
\hline 8 & 0.1893 & Pinche & 0.3078 & Joto \\
\hline 9 & 0.1851 & Joto & 0.2241 & Pícatelo \\
\hline 10 & 0.1835 & Verga & 0.2218 & Maricón \\
\hline
\end{tabular}

Las respuestas del lugar uno al cuatro de los centros educativos son idénticas en el cuadro comparativo anterior, lo que varía es la posición, por ejemplo, el vocablo 'puto' se ubica en centro privado en primer lugar y en el tercero en público; un compuesto sintagmático apareció en primer lugar en centro educativo público y tercero en privado. En el cuarto puesto, se estandarizó la muestra con el vocablo 'güey'. En relación con este vocablo, su uso en el medio social ha hecho que modifique el significado, en un primer momento peyorativo para después obtener el valor social-cortés. A partir de la quinta posición, la distribución es variada. Aparece en tercera posición 'chinga tu madre' para centro privado y en quinto y sexto lugar 'hijo de puta' y 'vete a la verga'.

También, en escuela pública, en el noveno lugar ha aparecido el vocablo 'pícatelo'. Su estructura hace referencia a 'verbo + PPÁ' y su significado es interpretado como una acción agresiva-física, totalmente distinta a la agresión verbal que muchas de las respuestas presentan. Este cambio de significado es común dentro del insulto, ya que pasa de la agresión verbal a la agresión física que se empieza a observar con los compuestos sintagmáticos. En este contexto, hacen su aparición vocablos como 'pinche', ‘joto', 'verga', 'maricón', etc., que generan un nuevo significado al insulto: el menosprecio y la intolerancia. Esto se aprecia con ‘joto’ o 'maricón'. Un vocablo como 'verga' no necesariamente pertenece al insulto, pues es una parte del cuerpo; sin embargo, esta parte siempre o casi siempre está vinculada al compuesto sintagmático 'vete a la verga' en relación con el insulto.

\section{Vocabulario - interacción}

Antes de abordar la relación disponibilidad léxica-socio-pragmática, y en la medida de las posibilidades, se tratará de observar cómo la posición de los vocablos disponibles producto 
de los IDL está correlacionada a la posición en que aparecen los vocablos en las interacciones. En este mismo tenor, las formas juveniles que se observan en el uso de los vocablos son propias de la edad, del sexo y de otras tantas variables que les permiten desarrollar su vocabulario para interactuar y, cuando lo hacen, tienden a ser directos y sinceros con otras personas, sin que por ello sientan remordimiento ni afectación a su imagen o a la de los otros por el uso de un lenguaje tabuizado y disfemístico (Zimmermann, 2002 en Ortega Salamanca y Vargas Cortés, 2017).

No obstante, mucho de este vocabulario tiende a ser agresivo, peyorativo o insultante, a pesar de que se disfraza en la interacción entre jóvenes que se produce de forma oral en conversaciones informales y espontáneas. Muchas de las conversaciones refuerzan el contacto social, en otras, la agresión verbal. Así pues, teniendo en cuenta lo anterior, vocablos como 'puta(o)', 'pendejo,, 'estúpido', 'güey', 'idiota', 'chinga tu madre', 'zorra', 'cabrón', 'hijo de tu pinche madre', etc., no es extraño escucharlos en conversaciones de este tipo.

Las correlaciones entre los vocablos disponibles y su posición en la interacción se deben, también, al efecto social que establece este centro de interés con la actividad comunicativa; es decir, las consecuencias de una actividad comunicativa agresiva como esta, reflejada en los vocablos disponibles y sus respectivas correlaciones, generan, sobre la idea de un clima socioemocional de la interacción y sobre la imagen social de la persona, un vocabulario altamente regular vinculado a la cantidad de respuestas activadas por la inercia comunicativa (Bravo, 2003). Así pues, esta inercia comunicativa es lo que permite mantener la estabilidad del vocabulario como las correlaciones, ya que al interrumpir o romper la comunicación, también se rompe la aparición de los vocablos con el resto de las otras posibles respuestas que pudieran activarse en esta inercia (Mateo y Yus, 2013). Una forma de hacer correlaciones y entender el comportamiento léxico es observarlo en las posiciones en que aparece en la interacción, esto ayuda a comprender la naturaleza léxica en la naturaleza interactiva del discurso. Es un proceso de decodificación y comprensión que se relaciona con el vocabulario disponible que se presenta en la misma interacción y que provee suficiente significado como para asociar las formas léxicas con algún contenido semántico que se encuentra en el acto de habla (Ortega Salamanca y Vargas Cortés, 2017, pp. 83-103). Como ejemplo de esto, se presenta la siguiente interacción de alumnos del centro educativo secundaria:

H1. ¡Óscar! ¡Qué chingaos dices de mí?

H2. Nada, güey ¿por qué?

H1. Pus dice el Benito que dijiste que yo era puto, porque no me gustan las viejas.

H2. Pus sí, güey, ¡qué! ¡a poco no es verdad!

H1. No güey, me gustan las viejas.

H2. No, pareces puto, un puto joto.

- (se acerca otro participante)

H3. Sí güey, eres un puto y bien pinche maricón.

H1. No, ustedes están pendejos, no soy puto.

H2. ¡Pendejos!, pendejo tú.

H1. Qué güey, la vas armar güey.

H2. Pus lo que quieras.

H1. A mí no me dicen puto, puto eres tú.

H2. Chinga tu madre, pinche maricón.

H3. Ya, lárguense par de maricas. 
Como se observa, la imagen individual del informante H1 se ve afectada cuando el informante $\mathrm{H} 2$ agrede no intencionalmente la afirmación del primero. Este tipo de agresión es evasiva, es decir, no acepta la culpa en primer momento de lo que el informante H1 menciona. La agresión a la imagen pública de H1 se ve afectada en la identidad social que se ve cuando H3 interviene; es decir, es proyectada a la apertura para que este informante intervenga. En la Tabla 8 se aprecia mejor la posición de los vocablos en la interacción:

Tabla 8. Posición de los IDL en la interacción de hombres

\begin{tabular}{|c|c|c|c|c|c|c|c|c|}
\hline $\begin{array}{c}\text { Turnos en la } \\
\text { conversación } \\
\text { H1. }\end{array}$ & IDL & Posición & $\begin{array}{c}\text { Turnos en la } \\
\text { conversación } \\
\text { H2. }\end{array}$ & IDL & Posición & $\begin{array}{c}\text { Turnos en la } \\
\text { conversación } \\
\text { H3. }\end{array}$ & IDL & Posición \\
\hline 1 & 0.0991 & 189 & 2 & 0.3185 & 4 & 7 & $\begin{array}{l}0.3185 \\
0.1813 \\
0.1121 \\
\end{array}$ & $\begin{array}{c}4 \\
17 \\
22 \\
\end{array}$ \\
\hline 3 & 0.1813 & 17 & 4 & 0.3185 & 4 & 13 & 0.2111 & 17 \\
\hline 5 & 0.3185 & 4 & 6 & $\begin{array}{l}0.1801 \\
0.1011\end{array}$ & $\begin{array}{l}22 \\
28\end{array}$ & & & \\
\hline 8 & $\begin{array}{l}0.2189 \\
0.1813\end{array}$ & $\begin{array}{l}14 \\
17\end{array}$ & 9 & 0.4242 & 2 & & & \\
\hline 10 & $\begin{array}{l}0.0945 \\
0.3185\end{array}$ & $\begin{array}{c}46 \\
4\end{array}$ & 12 & $\begin{array}{l}0.2855 \\
0.1121\end{array}$ & $\begin{array}{c}6 \\
22\end{array}$ & & & \\
\hline 11 & $\begin{array}{l}0.3983 \\
0.3983\end{array}$ & $\begin{array}{l}3 \\
3\end{array}$ & & & & & & \\
\hline
\end{tabular}

El informante H1 inicia la interacción con un vocablo que se ubica en la posición 189 del índice de disponibilidad. En cuestión de la clasificación propuesta de vocablos de apertura, estabilidad y cierre, se observa que 'puto' se ubicó en la posición 189 de aparición, cuando se analizan las conversaciones y se observa la variación en su IDL, este llega a la posición 17 y de esta a la cuatro, para luego subir a la posición 46 y estabilizarse en la posición tres. Es una posición en donde H1 cierra la intervención en la conversación. Este mismo patrón de H1 se observa también en $\mathrm{H} 2$ e, incluso, en H3. En H2 inicia con un vocablo que se ubica en cuarta posición, se mantiene, sube hasta una posición 28 , regresa al lugar dos, sube al seis y termina en el número 22 en la intervención 12. Como se aprecia, los vocablos disponibles siempre se encuentran en el desarrollo de la conversación activados y aparecen continuamente sin dejar de salir de la posición de estabilidad.

En H3, un informante que se suma a la conversación en la intervención siete, aparece con un vocablo que se ubica en la posición cuatro, producto de la relación que se dio en la intervención cinco, después va hacia arriba hasta alcanzar la posición 22 pasando por el lugar 17 y termina en este lugar en la intervención 13. Con lo anterior, se puede observar que la estabilidad del léxico disponible no solo se encuentra en las listas de disponibilidad que se obtienen cuando se utiliza un detonador conceptual, sino en la misma posición dentro de una interacción lingüística. En la conversación que se analizó, se aprecia cómo el vocabulario de este centro siempre tiende a mantenerse entre los niveles de respuesta más elevados, lo que significativamente establece una correspondencia entre el IDL y la posición del vocablo en esa 
interacción. Esto se comprueba a continuación cuando se ubica el IDL y la posición en la que apareció el vocablo en la interacción ${ }^{4}$ de varones.

1) H1. Qué chingaos $(0.0991,189)$

2) H2. Güey $(0.3185,4)$

3) H1. Ser puto $(0.1813,17)$

4) H2. Güey $(0.3185,4)$

5) H1. Güey $(0.3185,4)$

6) H2. Parecer puto $(0.1801,22)$, puto joto $(0.1011,28)$ - (se acerca otro participante)

7) H3. Güey $(0.3185,4)$, ser puto $(0.1813,17)$, pinche maricón $(0.1121,22)$

8) H1. Estar pendejos $(0.2189,14)$, ser puto $(0.1813,17)$

9) H2. Pendejos $(0.4242,2)$, pendejo $(0.4242,2)$

10) H1. Qué güey $(0.0945,46)$, güey $(0.3185,4)$

11) H1. Puto $(0.3983,3)$, puto $(0.3983,3)$

12) H2. Chinga tu madre $(0.2855,6)$, pinche maricón $(0.1121,22)$

13) H3. Maricas $(0.2111,17)$

Cuando se obtienen los resultados de la encuesta de disponibilidad, el acumulado y la posición en el que se dijo esa respuesta, se aprecian mejor las distribuciones de los vocablos y su resultado determinará su frecuencia en función de las ocasiones en que ha sido utilizado ese vocablo en la interacción. La suma de las repeticiones determinará si pertenece a las respuestas más disponibles. En ese sentido, las correlaciones entre el IDL y el vocabulario disponible son como un nexo natural que se ve reflejado en cada una de las interacciones, y aflora cuando el sujeto activa en la interacción un determinado tema. Como ejemplo de esto, se presenta, de nueva cuenta, otra conversación, ahora de mujeres.

M1. Pinche Luis, me dejó.

M2. Ese culero, siempre fue así, es un ojete.

M1. Sí güey, ese güey nada más jugó conmigo.

M2. Anda ahora con la Daniela.

M1. Ese hijo de su pinche madre se burló.

M2. Sí, que te parece si vamos y le rompemos la madre a la Daniela.

M1. Sí, pero, ahora que recuerdo, esa vieja anda con las abejas negras y sabes que son pinches viejas pelioneras.

M2. Entonces ¿qué hacemos?

M1. No sé, ese cabrón me las va a pagar.

M2. ¿Cómo?

M1. No sé güey.

Tal como se ha explicado, las correlaciones léxicas pueden generar información relevante de la posición que guardan los vocablos en una interacción real, y cuando se analiza el léxico disponible del lenguaje juvenil, se debe considerar por un lado la variedad léxica de los mismos informantes y por el otro, la capacidad de generar nuevos términos para enriquecer el lenguaje coloquial con el que se comunica. Así pues, lo importante de analizar

4 En el paréntesis se aprecia el IDL y la posición en que apareció el vocablo en la lista. 
las correlaciones léxicas por IDL y por uso en el texto, permitirá observar el cambio lingüístico latente que está en la lengua. A continuación, se presentan los IDL y la posición de los vocablos de la conversación de mujeres de secundaria.

1. M1. Pinche $(0.2033,8)$

2. M2. Culero $(0.1598,19)$, Ser ojete $(0.0992,27)$

3. M1. Güey $(0.3185,4)$, güey $(0.3185,4)$

4. M2.

5. M1. Hijo de su pinche madre $(0.1785,9)$

6. M2. Romper la madre $(0.08261,30)$

7. M1. Pinche $(0.2033,8)$

8. $\mathrm{M} 2$.

9. M1. Cabrón $(0.2118,7)$

10. M2.

11. M1. Güey $(0.3185,4)$

En la conversación -y a diferencia de la conversación de los varones-, hay menor uso de vocablos que designan insulto. Son ocho vocablos que han aparecido distribuidos, como se observa en la siguiente Tabla 9:

Tabla 9. Posición de los IDL en la interacción de mujeres

\begin{tabular}{|c|c|c|c|c|c|}
\hline $\begin{array}{c}\text { Turnos en la } \\
\text { conversación } \\
\text { M1. }\end{array}$ & IDL & Posición & $\begin{array}{c}\text { Turnos en la } \\
\text { conversación } \\
\text { M2. }\end{array}$ & IDL & Posición \\
\hline 1 & 0.2033 & 8 & 2 & $\begin{array}{r}0.1598 \\
0.0992\end{array}$ & $\begin{array}{l}19 \\
27\end{array}$ \\
\hline 3 & 0.3185 & 4 & 6 & 0.0826 & 30 \\
\hline 5 & 0.1785 & 9 & & & \\
\hline 7 & 0.2033 & 8 & & & \\
\hline 9 & 0.2118 & 7 & & & \\
\hline 11 & 0.3185 & 4 & & & \\
\hline
\end{tabular}

Nótese que el informante uno aporta mayor cantidad de vocablos disponibles a la interacción. En palabras de Rojas, Zambrano y Salcedo (2017, pp. 1-13), es muy común que en una interacción el informante que inicia la interacción sea quien aporta mayor cantidad de vocablos, aunque en algunos casos no sea así, más cuando se refiere a interacciones donde existen varios participantes. No obstante, en esta se aprecian las posiciones de los vocablos: inicia en la posición ocho, baja a la cuatro, sube a la nueve, regresa a su posición inicial para mantenerse antes del cierre con la posición cuatro. En este caso, la segunda posición (cuatro) se repite al cierre. Estas son las correlaciones entre el léxico disponible y la posición que guardan al interior de la conversación. En lo que refiere a la participante dos, las posiciones de interacción son altas y se debe a que no participa en la conversación, sino simplemente mantiene el diálogo, lo que genera posiciones muy alejadas de los diez lugares más disponibles $(19,27$ y 30). Tal como se aprecia, en la cultura juvenil, el uso de este vocabulario forma parte de las conversaciones coloquiales y su aparición está vinculada a expresiones malsonantes y peyorativas que van activándose de acuerdo con la intencionalidad; aunque en este trabajo no se 
analice esta intencionalidad, sí se puede apreciar la manera cómo el vocabulario utilizado ocupa las posiciones con los IDL más elevados. Ortega Salamanca y Vargas Cortés (2017) sostienen la idea de que, en el contexto escolar, se observa que uno de los rasgos lingüísticos más relevantes en las interacciones comunicativas de los jóvenes es el uso y la aceptación de expresiones que, desde otros escenarios, son considerados como descorteses y denigrantes de la imagen social.

\section{Conclusiones y discusiones}

En este trabajo se ha tratado de generar un vínculo entre la disponibilidad léxica y la socio-pragmática a través del análisis del centro de interés del insulto y, para ello, se ha analizado el vocabulario que se utiliza para este fin. No obstante, se ha observado que el adjetivo es el tipo de vocablo que más aparece en el análisis. Como se aprecia, en un primer momento se había pensado que este centro sería muy difuso por el vocabulario recogido y analizado; sin embargo, se ha observado que dentro de las diez respuestas más disponibles se encuentran cuatro adjetivos, dos nominales y cuatro compuestos sintagmáticos que aparecen de manera repetitiva en las interacciones analizadas, por lo que este centro de interés, al igual que otros centros, es compacto. En lo referente a los adjetivos 'puta', 'pendejo', 'puto' y 'estúpido', estos corresponden a las respuestas más disponibles de la muestra, si se considera el número total de vocablos que asciende a 356 vocablos de 6489 palabras, y son vocablos que se pueden combinar para formar compuestos sintagmáticos como 'puta perra' o 'Juan pendejo', que aparecieron en las últimas posiciones de los vocablos. Los adjetivos son la categoría gramatical que más aportación ha hecho a este centro con 159, le siguen los compuestos sintagmáticos con 129 y, en último lugar, 68 nominales que aparecieron en los resultados globales. Sin duda alguna, este centro debe clasificarse en los centros de interés que obtienen adjetivos como respuestas disponibles, entre los que destacan los centros de interés "Los colores", "Los adjetivos", "Los adjetivos calificativos", "Aspecto físico y carácter de los españoles y portugueses", entre otros. En lo que refiere a los compuestos sintagmáticos, se encuentran 'chinga tu madre', 'hijo de tu pinche madre', 'pinche culero', etc. Son las respuestas prototípicas de este centro y son las que más se usan en las interacciones, tal como se ha visto en las conversaciones analizadas.

En lo que se refiere a las correlaciones, se puede apreciar que los hombres usan más las groserías como elementos de agresión que las mujeres, lo cual se debe de cierta manera a factores sociales que se establecen en el ámbito laboral, es decir, el hombre al convivir con otros hombres utiliza un discurso prototípico que tiene que ver con el uso de las groserías como marca de distinción entre hombres y mujeres; en cambio, socialmente se ve mal que la mujer use este tipo de discurso frente a la sociedad, por ende, su forma de expresión es más elaborada y menos soez o vulgar que la de los hombres. De las correlaciones que se obtuvieron entre el vocabulario y su aplicación, se ha observado que la estabilidad del léxico está vinculada con la activación del centro de interés. Los vocablos de apertura activan la información del centro de interés al principio de la interacción, después de unos intercambios comunicativos, estos vocablos alcanzan su estabilidad en el discurso, generando la aparición de los vocablos disponibles que el informante usa para comunicarse.

Por último, se ha podido observar que sí existe una correlación de los vocablos obtenidos de los cuestionarios con los lugares que ocupan en las conversaciones. Es relevante si se considera que este vocabulario se obtiene cuando se estimula al informante a través de un 
centro de interés. En este caso, no solo se visualizaron las respuestas, sino que se observaron en las interacciones que no fueron sencillas de obtener por lo complejo que resultaba hacer las grabaciones. Sin embargo, el contar con estos primeros resultados permitirá abrir un nuevo campo de investigación que es fundamental si se quiere que los estudios de disponibilidad léxica tengan mayor impacto en otras áreas del saber lingüístico.

\section{Bibliografía}

Bravo, D. (2003). Actividades de cortesía, imagen social y contextos socioculturales: una introducción. En D. Bravo (Ed.), Actas del Primer Coloquio Edice (pp. 98-108). Estocolmo: Stokcholms Universitet.

Brenes Peña, E. (2007). Estrategias (Des)Corteses y Agresivas en la Figura del Tertuliano Televisivo: ¿Trasgresión o Norma? Lingüística en la Red, 5, 1-19.

Calsamiglia, H. y Tusón, A. (2002). Las cosas del decir. Manual de análisis del discurso. Barcelona: Ariel.

Cava, M. J. y Musitu, G. (2002). La convivencia en la escuela. Barcelona: Paidós Ibérica.

Cepeda, M., Cárdenas, Á., Carrasco, M., Castillo, N., Flores, J., González, C. y Oróstica, M. (2017). Relación entre disponibilidad léxica y comprensión lectora, en un contexto de educación técnico profesional rural. Revista Sophia Austral, 1(1), 81-93. Recuperado de http://www. sophiaaustral.cl/index.php/shopiaaustral/ article/view/51/46

Cerezo, M. A., Dolz, L., Pons-Salvador, G. y Cantero, M. J. (1999). Infancy Maltreatment Prevention: A Program Impact on Child Development. Anales De Psicología / Annals of Psychology, 15(2), 239-250. Recuperado de https://revistas.um.es/analesps/article/ view/30131

Gómez Molina, J. R. (2000). El insulto en la interacción comunicativa. Estudio sociolingüístico. Oralia, 4, 103-132.

Hernández Muñoz, N. (2006). Hacia una teoría cognitiva integrada de la disponibilidad léxica: El léxico disponible de los estudiantes castellanos-manchegos (Tesis de grado). Universidad de Salamanca.

INEGI. (2018). Encuesta Nacional de Victimización y Percepción sobre Seguridad Pública. Instituto Nacional de Estadística y Geografía. Recuperado de https://www.inegi.org. mx/programas/envipe/2018/

Lakoff, R. T. (1973). The Logic of Politeness, or Minding your P's and Q's. En C. Corum, T. C. Smith-Stark y A. Weiser (Eds.), Proceedings of the Ninth Regional Meeting of the Chicago Linguistic Society (pp. 345-356). Chicago: Chicago Linguistic Society.

López Chávez, J. y Strassburger Frías, C. (1991). El diseño de una fórmula matemática para obtener un índice de disponibilidad léxica confiable. Anuario de Letras. Lingüística y Filología, 38, 227-251.

López Morales, H. (1995). Los estudios de disponibilidad léxica: pasado y presente. Boletín de Filología, 35(1), 245-259. Recuperado de https://revistaderechoambiental.uchile.cl/ index.php/BDF/article/view/19231/20354 
López Rivero, E. (2008). Estudio de disponibilidad léxica en 43 estudiantes de ELE. Memoria de máster en Enseñanza del Español como Lengua Extranjera, Universidad Antonio de Nebrija. Recuperado de http:/www.educacionyfp.gob.es/educacion/mc/redele/ biblioteca-virtual/numerosanteriores/2008/memoriamaster/2-semestre/lopez-r.html

López, C. y Torner, S. (1992). Disponibilidad léxica y ponderación en el discurso académico. El uso de los adjetivos en el Corpus 92. Revista de Estudios de Adquisición de la Lengua Española, 11, 23-46.

Martínez Lara, J. A. (2009). Estudio sociopragmático del uso del insulto en la comunidad juvenil universitaria (Tesis de grado). Universidad Central de Venezuela.

Mateo, J. y Yus, F. R. (2013). Towards a cross-cultural pragmatic taxonomy of insults. Journal of language aggression and conflict, 1(1), 87-114.

Ortega Salamanca, F. J. y Vargas Cortés, B. (2017). Aproximación sociopragmática a las estrategias conversacionales de los adolescentes. Cuadernos de Lingüística Hispánica, 29, 83-103.

Ramírez Fuenanta, C. (1999). Conductas agresivas en la edad escolar. Madrid: Pirámide.

Rey, R. del y Ortega, R. (2008). Bullying in poor countries: Prevalence and coexistence with other forms of violence. International Journal of Psychology and Psychological Therapy, 8(1), 39-50.

Rojas, D., Zambrano, C. y Salcedo, P. (2017). Metodología de análisis de disponibilidad léxica en alumnos de pedagogía a través de la comparación jerárquica de lexicones. Formación Universitaria, 10(4), 1-13.

Ruiz, G. y Muñoz, G. (en prensa). Acoso escolar. En J. Sanmartín, J. R. Gutiérrez Lombardo y J. Martínez Contreras (Eds.), Reflexiones sobre la violencia.

Samper-Hernández, M. (2009). Evolución de la disponibilidad léxica en estudiantes grancanarios de enseñanza primaria y secundaria. Las Palmas de Gran Canaria: Cabildo de Gran Canaria.

Searle, J. (1990). Actos de habla: ensayo de filosofía del lenguaje. Madrid: Cátedra.

SEP. (2017). Estadística del Sistema Educativo de San Luis Potosí, ciclo escolar 2016-2017. México: Secretaría de Educación Pública. Recuperado de http://snie.sep.gob.mx/ descargas/estadistica_e_indicadores/estadistica_e_indicadores_educativos_24SLP.pdf

Smith, P. K. (2004). Bullying: recent developments. Child and Adolescent Mental Health, 9(3), 98-103.

UNICEF (2019). Violencia y maltrato. Recuperado de https://www.unicef.org/mexico/spanish/ proteccion_6932.htm

Wingeyer, H. R. (2014). Temas de la disponibilidad léxica de la región NEA de argentina: mitos populares, la ciudad, el campo, insultos y diversiones nocturnas. Aportes para la elaboración de programaciones interculturales y de materiales en la enseñanza de E/ LE. Signos ELE. Revista de español como lengua extranjera, 8, 1-14.

Zimmermann, K. (1996). Lenguaje juvenil, comunicación entre jóvenes y oralidad. En T. Kotschi, W. Oesterreicher y K. Zimmermann (Eds.), El español hablado y la cultura oral en España e Hispanoamérica (pp. 475-514). Madrid: Iberoamericana. 